\title{
New Landscapes and New Eyes: The Role of Virtual World Design for Supply Chain Education
}

\author{
Theo J. Bastieans, Fernuniversität Hagen, Germany \\ Lincoln C. Wood, Auckland University of Technology, New Zealand and \\ Curtin University, Australia \\ Torsten Reiners, Curtin University, Australia
}

\begin{abstract}
With the common availability of advanced educational technology, we are able to increase the emphasis on the design of learning experiences and benefit from the given flexibility and variety of opportunities to create learning spaces. As instructional design models become more commonplace we examine their role vis-à-vis with the fidelity of the experience while learning. Highfidelity experiences are known to be valuable in learning as they provide authenticity in learning and motivation; yet, high fidelity comes at the cost of greater investment. In this paper we outline our experiments with two setups of differing levels of fidelity: using Second Life and the consumer-focused Oculus Rift Head-Mounted Display (HMD). We show qualitatively interpreted comments and user responses to demonstrate importance of the level of fidelity, uncover important elements, and relate back the fidelity to the learning experience. High-fidelity experiences can be supported by software and hardware that are now readily available but present the seductive opportunity to greatly improve participant engagement in the virtual environments presented.
\end{abstract}

Keywords: Instructional Design, Immersion, Virtual Reality, Second Life, Oculus Rift, Head-Mounted Displays (HMD), Fidelity

"The real voyage of discovery consists not in seeking new landscapes but in having new eyes." Marcel Proust (French Novelist and Author, 1871-1922).

As already described in the year 2000, conventional teaching and learning can be an excellent form of education but many times it is disappointing with outcomes falling short of expectations (Bastiaens and Martens 2000). Most forms of conventional education have an emphasis on the supporting role of teachers, although there are quite a number of educational objectives that cannot be achieved by just focusing on the supporting role. A teacher can support students and can convey expert knowledge but it is the student himself who must get engaged in learning by working on learning tasks, e.g., reading, understanding, and memorising. Moreover, the advantages associated with conventional education are largely irrelevant when it comes to certain subjects. Self- and independent-study is often an equally good or even better method of attaining learning goals, especially at an 'advanced' level (Bastiaens and Martens 2000, 4). Another important drawback to conventional education is the dependence on the quality and the motivation of the individual teacher and the size of the class. More individualised learning in a well-constructed educational environment can offer a constant high-quality experience to learners.

In this paper we discuss virtual and authentic learning environments as extremely malleable spaces, that can be used as a 'functional' space (i.e., with connected processes located 
close by) and have a 'realistic' representation of the world (i.e., with real-world mimicry of locations, geography, and infrastructure which has an impact on firms in the supply chain). Of primary importance is not only that environments are used, but guidelines support the how this can be done in instructional design. Clearly, creation of the environment requires resourcing and pertinent questions like:

- What level of fidelity or realism is appropriate to motivate students?

- What elements are important in a high-fidelity experience?

After a review of relevant literature discussing instructional design, virtual environments, and immersion, we present our methodology and two experimental setups of differing levels of fidelity. The experimental findings are related to key constructs.

\section{Literature Review and Theoretical Considerations}

For several years, it has been argued that rapid technological and societal changes result in a need for workers to possess complex knowledge. As a result, perspectives on learning are changing to ensure that graduates' capability match employers' requirements. Solving real-world problems and dealing with complex skills have become the main goal in teaching. Modern instructional design (ID) models assume that realistic and rich learning tasks are the driving force for learning (Merrill 2002). Well-designed learning tasks encourage learners to integrate and coordinate the required skills, knowledge, and attitudes (Deimann and Bastiaens 2010). The term 'competence' has emerged as a major concept to describe this process. While there are several definitions, competencies can be described as context-based cognitive dispositions that are functionally related to situations and demands in specific domains (Klieme and Leutner 2006). In competency-based learning, learners are no longer primarily trained to pass their examinations but also to learn independently and to manage their own learning process (Deimann and Bastiaens 2010). Learning occurs when learners are engaged with authentic learning tasks, delivering a learning experience closely matched to reality; active learning with engagement in activities can also support a wider range of learners (Wood and Reefke 2010). Competencybased learning is seen as the way in which to respond appropriately to the claims and demands of business and industry, and to prepare learners for the role they will play in the future of working life and society. A competence developed and assessed in a more authentic learning task is more likely to be transferable and valuable into the working environment. This authenticity is a crucial element that underpins the learning experience.

Virtual learning environments can provide the opportunity to build on these ID Models and advance them further, which can warrant continuous high quality in teaching and learning. The Four-component Instructional Design Model developed by van Merriënboer (1997) offers designers guidelines for the development of complex and authentic learning experiences and consists of four interrelated blueprint components (Figure 1). 'Learning tasks' form the backbone of the model; they are defined as concrete, authentic, and meaningful 'whole task experiences'. The second component is called 'supportive information'. This is information that is supportive to the learning and performance of non-recurrent aspects of learning tasks; e.g., problem solving and reasoning. 'Just-in-time information' is the third component; it is a prerequisite to the learning and relevant to the performance of recurrent aspects of learning tasks. In general, these 
are small information units that are presented to learners in a 'just-in-time' mode while they are working on the learning tasks. The last component is called 'part task practice' being additional exercises for recurrent aspects of learning tasks for which a very high level of automation is required (after instruction).

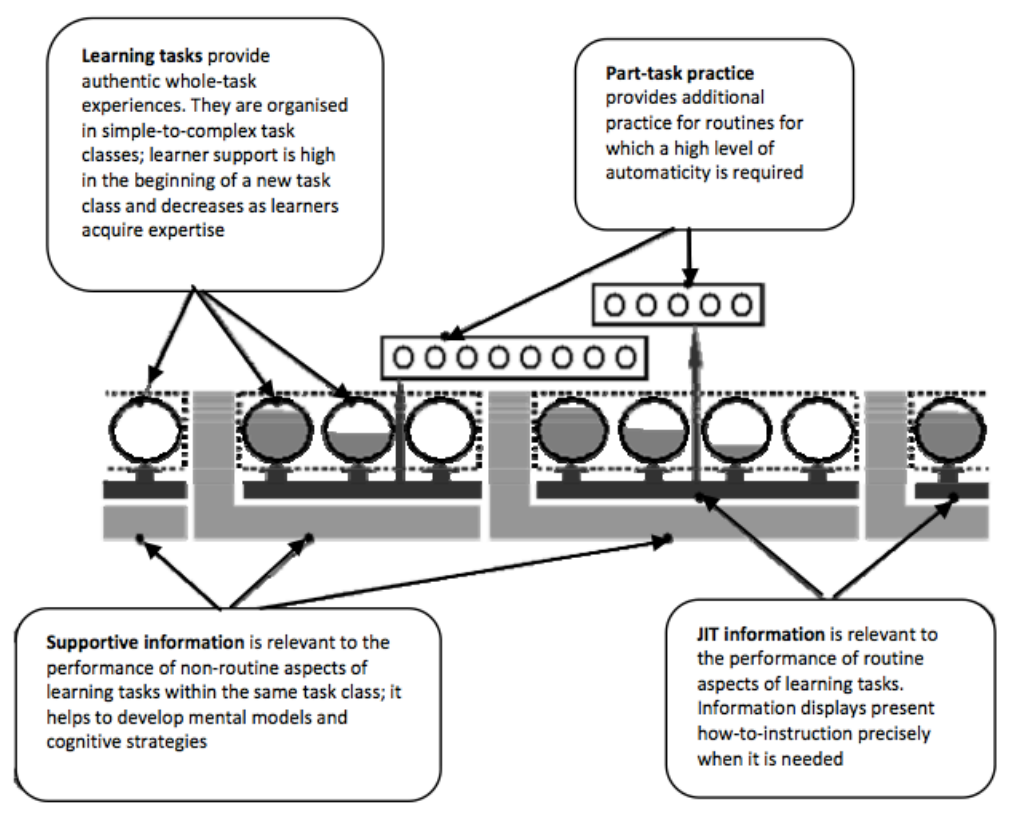

\section{Figure 1. A schematic overview of the four components of the Instructional Design Model, adapted from Van Merriënboer, Bastiaens, and Hoogveld (2004, 15)}

Working on authentic learning tasks improves the transfer of learning to other situations; later, learners recognise similarities in their jobs to the authentic tasks they were exposed to during training, making it easier to reconnect to learned skills or knowledge. When we consider the resources required to create entirely new and highly authentic learning tasks we stumble on the following question, which is driven by emerging cost-sensitivity in most educational environments: How much authenticity is 'enough'? This is an important question as we still have little understanding about the level of authenticity and realism required within such environments to sufficiently deliver the learning material. Gulikers, Bastiaens, and Martens (2005) showed that learning tasks and the learning environment do not necessarily need to be 'high fidelity' for improved learning results. High fidelity means that the environments resembles real life to a high degree or duplicates it (i.e., if a very realistic simulation is used). Low fidelity environments and tasks are frequently good enough. This means that a learning environment and tasks must meet the same tasks criteria (i.e., the same output and results are expected), the same social context (is the task in cooperation with certain stakeholders or not) and the same physical objects (which objects are available in normal life).

This result is counter-intuitive; surely, we would say that a more realistic scenario is better! High fidelity may have distinct advantages. One of these is the depth of immersion that the learner can feel within the experience; immersion means that "the greater the participant's suspension of disbelief that she or he is 'inside' a [...] setting" (Dede 2009, 66) the more immersed they are. Distinctly, there is perceptual immersion: "the degree to which a virtual 
environment submerges the perceptual system of the user" (Biocca and Delaney 1995, 57) and psychological immersion, the degree of feeling of involvement and enthralment caused by stimuli (Palmer 1995). High fidelity can be connected to perceptual immersion while psychological non-immersion may be caused. Thus, low fidelity (i.e., low levels of perceptual immersion) scenarios may still have a high degree of psychological immersion. It is often this psychological immersion that is connected to the higher levels of authenticity in the learning tasks. However, some moderate degree of fidelity is likely to be required. But how much? The greater the fidelity the greater the perceptual immersion is likely to be, and therefore, the greater the learning.

High fidelity, incorporating head-mounted displays (HMD) and sound, creates a truly intense experience that allows participants to take scenarios more seriously, increasing the authenticity of learning tasks. Note, that while 'authenticity' is often assumed to imply 'realistic', it is instead most important that the learning and activities reflect the development and use of the knowledge as required in the given real or virtual environments (Herrington et al. 2010). Such a process creates improved transferability as the participants increasingly take the scenario for real. Thus, a process can be highly authentic, but in a non-realistic setting. For example, if you feel that you are really on the edge of a building or the Grand Canyon, you are likely to approach the edge with caution; despite the level of realism with respect to the computer graphics. Similarly, learners are more likely to take a health and safety training program more seriously and treat it with greater respect as they seek to understand and learn, aware that the experiences must be taken seriously.

Immersion in the virtual environment is crucial to gaining learner engagement in the educational experience. This has been demonstrated in other settings and can be supported through elements such as virtual bots, structured activities, narratives, inter alia. Here we look at one element that is, in our opinion, often overlooked: the 'virtual terrain' of the world and learning environment. This is often crucial in many business settings, particularly in logistics and supply chain management, where it makes a significant difference to real-world supply chain operations. In these educational environments, sensibly scoped spaces are required to 'set the scene' for the students.

Perceptual immersion means more than simply 'seeing' what is going on in the world around you; it involves senses other than sight. Yet, the more senses we stimulate in a simulation, the more expensive and complex the required equipment becomes. Immersion, particularly perceptual immersion, can be further enhanced through the use of technologies such as haptic feedback (e.g., the use of a vibration that coincides with a collision), virtual-reality (VR) gloves or visual-sensing technologies such as Kinect to improve control, or the use of head-mounted displays such as the Oculus Rift set. Together, these submerge the user into a virtual realm of intense fidelity. Caves, specialised 3D simulation rooms, are enclosed spaces that respond to the movement of the users and update projections around them. Caves use specialised equipment that is expensive, occupies valuable space, and are challenging to install and maintain in use. However, this "room-sized, walk-in virtual reality [...] display is to a typical computer screen what a supercomputer is to a laptop computer" (DeFanti et al. 2009, 169). More suitable for home-based users and vastly cheaper and therefore more accessible, are HMDs that use LCD screens in front of the eye, allowing a user to evaluate a different perspective with a turn of the head and see the image updated accordingly. Depth scanners used by Kinect can identify movements that can be used as a control mechanism, while the Razor Hydra tracks movements 
of the hand holding it. Our use of the concept of 'fidelity' in this research also encompasses the realism and fluidity of interaction with the environment. Using Second Life on a traditional computer monitor, interacting with keyboard and mouse, is of vastly lower level of fidelity than the experience in a Cave; 'holodeck' in Star Trek; the virtual environment in Matrix; or the visual-sensory integration used in Avatar (Apostolopoulos et al. 2012). However, educational settings often require high levels of functionality, but are motivated by limited resourcing. This may reduce both psychological and perceptual immersion in learning tasks. The 'sweet spot' must be identified where adequate resources allow creation of suitably immersive materials that relatively easily constructed and developed to gain the outcome and perceptual immersion with minimal effort; see Figure 2.

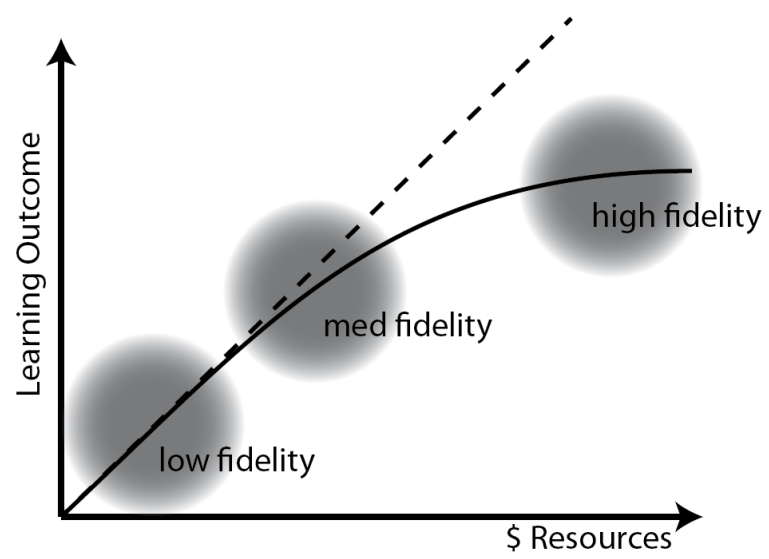

fidelity

Figure 2: Relation between resources and learning outcome regarding

In general, it is expected that learning in a high-fidelity and authentic virtual learning environment results in higher performance and improves intrinsic motivation of students. The questions are: where do we draw the line and say that adequate fidelity has been incorporated to achieve our outcomes without adding undue cost and expense in development and maintenance of the environment? What level of fidelity is appropriate to motivate users? What elements are important in the high-fidelity experience?

\section{Methodology and Implementation}

To increase our understanding of how the level of fidelity in virtual spaces relates to learning, a series of experiments were undertaken using different 'landscapes' and visualisation technologies. The research objective was to understand fidelity and perception within the virtual environment relative to motivation and interest of the students.

Students, academics, instructional designers, and innocent bystanders were invited to participate in the experiment. We ensured a range of backgrounds and interest amongst the 13 individuals that accepted the invitation to join the experiment. All participants expressed interest in virtual environments and learning more about emerging technologies in this area. The small sample size was judged adequate given the early stages of the research; a bigger sample at a later stage is required to show how specific characteristics of the participants (e.g., demographical, gender, age, education, and hobbies) affect the results. 
Six experiments were planned with different levels of immersion and fidelity, based on combinations of underlying 3D virtual world technologies and perception technologies. Specifically, Second Life was compared to the Unity 3D game engine and a normal computer monitor was compared to the Oculus Rift HMD. Following the participants interaction in the scenarios, short interviews were conducted and questionnaires were completed by the participants. Interviews were semi-structured and used open-ended questions. Likert-type scales with five levels were used in the questionnaire to rate and evaluate scenarios. A member of the research team was on-hand to record comments and make notes on gestures or interesting events during the experiments. Only the participant and experimenter were in the room to focus attention on the experience. Participants had restricted time within each scenario but were not rushed through, enabling them to explore the 3D spaces for a while. Approximately six minutes of experience per experiment was taken, with each participant involved for approximately 40 minutes.

We used two experimental setups. The first setup was a regular computer monitor running Second Life. The participants used the keyboard to control movements relative to the environment and the mouse to change the perspective. This was designed to be the low-fidelity environment. For the second setup we intended to create a higher fidelity environment; we opted to use the Oculus Rift HMD to support this. The technology development was initiated by a Kickstarter project designed to "revolutionize the way people experience interactive content" (Oculus 2013) through the use of consumer-oriented HMDs. The Oculus Rift matches images with head tracking using highly sensitive sensors, allowing near real-time update of images without any blind spots. The device is still under active development and has a resolution of 640x800 pixels per eye (in contrast to standard monitors with resolutions above 1600x900 pixels). The Oculus Rift HMD has been shown to present a significantly more realistic and compelling virtual world experience in comparison to traditional computer monitors (Reiner et al. 2014). While Reiners et al. (2014) report on the immersion achieved with the Oculus Rift HMD, we focus on examining the importance of fidelity experienced in the environment. We conducted the following experiments:

Experiment 1. Second Life was used. A normal monitor and keyboard were used by the participants to control their activities. They were asked to move towards a forklift, use the forklift, and drive around the warehouse; see Figure 3. The scenario included a short introduction about control in Second Life for those that had not used it before.

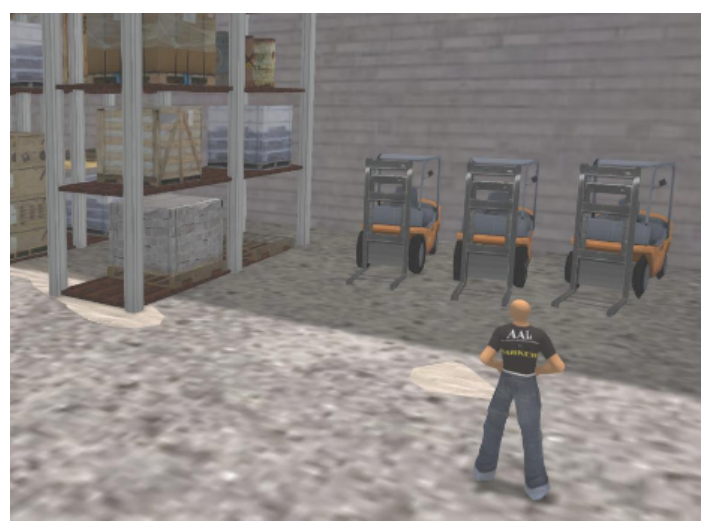

Figure 3: A user in the warehouse scenario 
- Experiment 2. The Oculus Rift 'House' scenario was used (http://sixense.com/sixensetuscanydemo where it is illustrated with the integration of Razor Hydra). While this is not directly comparable to the Second Life warehouse scenario, there were few Oculus Rift scenarios available due to the early development stages of the Oculus Rift HMD. The House scenario was considered comparable to Second Life but using the HMD with true 3D visualisation with control via keyboard; the shift of setting was considered less important than the 3D experience.

- Experiment 3. The Oculus Rift 'Roller Coaster' scenario has all movement controlled by the computer, with the user being able to look around to perceive what is happening in the 3D space. This scenario tested a larger space and evaluated how the participants reacted to the virtual roller coaster track.

- Experiment 4. A self-driving experiment on the 2D monitor. Here, the participants drove themselves around a virtual city.

- Experiment 5. Similar to experiment 4, but using the Oculus Rift for 3D visualisation (see http://www.decane.net/game/oculus-rift/riftracer). The participants were able to control and drive their vehicle themselves; see Figure 4.

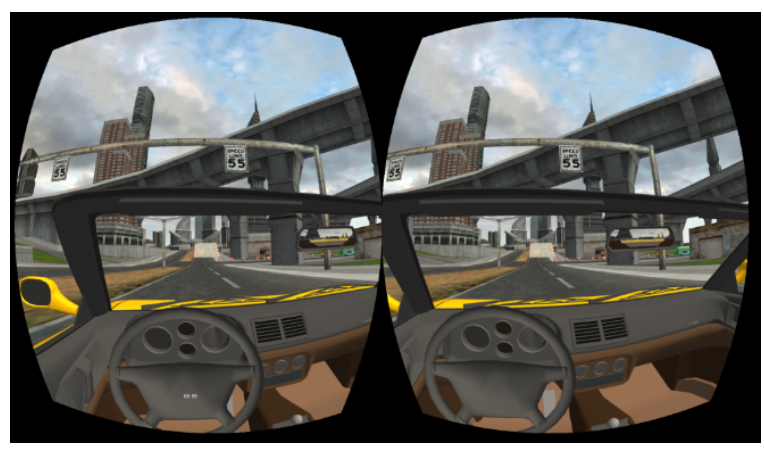

Figure 4: Driving simulator

- Experiment 6. The Oculus Rift 'Car Driving' scenario but controlled by the experimenter. This is similar to Experiment 3 where the participant has no direct control over the movement in the environment; yet they could look around without limitations.

The scenarios differ in terms of the level of fidelity employed in each. Second Life scenarios contained a realistic representation of real environments. It can be modified and adapted, with users creating new items and artefacts to populate this world. In the experiments, a traditional computer monitor (22 inch) was used with keyboard and mouse to control interaction with the environment. Oculus Rift scenarios were run using the Unity 3D engine. This has more visual 'special touches' than Second Life has (e.g., leaves that fall softly to the ground) and more realistic reflections in puddles of water. Benefits are gained by the use of the HMD, allowing a much more fluid immersion in perceiving the environment. However, a keyboard was used to interact with the environment, leaving room for higher fidelity experiences in future experiments. (Note that the keyboard is used to control movement in relation to the environment, while in Second Life also the mouse was used to control the viewpoints; clearly, a mouse is no longer required when using the HMD as the viewpoint shifts with a turn of the head.)

\section{Results}


Interaction when using the Oculus Rift HMD was more natural and extremely realistic, engaging, and compelling (Reiners et al. 2014). In short, participants described their HMD experience in situ using variations on these themes: "mind-blowing"; "sh*t, this is real", "oh my god", and "sick" (used in the colloquial sense to mean 'really great', 'cool', or 'crazy'). The following list shows the outcomes for different categories:

Detachment. The experiences with Oculus Rift HMD led participants to comment on their level of 'detachment' from the real world which led them to experience a period of reorientation when they finished their session with the HMD. While in session, they felt that it was almost 'too real'; it seemed to take them some time to realise that they were actually 'there'. However, another participant noted that they felt 'disconnected' from reality, but at the same time felt as though they were 'observing' in the virtual environment. Many participants attempted to 'grab' structures that existed in the virtual environment but did not exist in the real world; e.g., one participant attempted to grab the rails on the rollercoaster in Experiment 3. However, the level of detachment varied; one participant reported feeling 'teleported' to another place entirely with a complete severance of connection with the real world that was only reestablished with the researcher's voice.

Emotion. There were a range of emotions experienced. Notably, one participant said that they actually felt quite 'lonely' as the experimental scenarios did not include other people or other avatars; thus, their experience was not enriched as there was no social interaction.

Control of interaction. Participants preferred having only the keyboard to manage when using the HMD. However, they noted that it would be good to have further sensors to get their input or to move without the keyboard (e.g., by using a joystick). The use of keyboard made the experience 'less real'. From this we can see that greater fluidity and naturalness in the control systems would increase the fidelity further.

Ability to change perspective. Despite the ability to change perspective with a turn of the head using the HMD, many participants seemed to be 'stuck' in the mind-set of still looking 'straight ahead' just as they would when using a computer monitor. Only a couple seemed to make use of the ability to crane their heads around to see what was around them (particularly on the rollercoaster in Experiment 3).

Sensory perception. Sound was widely credited with adding to the experience as it was an important 'part of the illusion'. Only one participant noted otherwise and claims that sound wasn't related to their level of immersion; interestingly, this was a game-player who used extensive 'voice chats' while engaged in Counter-Strike and may therefore be desensitised to noises that may interfere with their perception of the reality of the environment. One participant noted that he could actually smell smoke from burning barrels while driving in the city - an interesting sensory hallucination.

Touch sensation. One user noted that it is unfortunate that they could not experience sensory feedback such as a physical 'bump' when they collided with a wall. Many participants attempted to physically grab or interact with something that was in the virtual environment. One participant, while driving, actually reached out to put their arm around the passenger seat when they went to reverse the vehicle. Some participants noted the incongruence created by their inability to actually feel the objects that they could 'see' in the environment. Contrariwise, some commented on the fact that they could not see their own body when they looked downwards. (A 
later user of the HMD, not in this experiment, also narrowly avoided a head injury when he decided to look down while forgetting that this motion was prevented by the table top under his head.)

Height or high points. One participant was noted to demonstrate extreme caution when near a high point on one scenario (Experiment 2). A quick conversation revealed no nervousness or fear of heights. Most participants did treat these 'high areas' with greater caution while using the HMD. This indicates a greater level of affinity and a higher level of fidelity from the experience.

Visual. While the display quality does not contain as many pixels as a full HD monitor, this was not considered to be a problem by most participants.

\section{Discussion and Application}

This research has indicated that the perceptual immersion and high fidelity of using the Oculus Rift HMD present valuable new opportunities for educators. The use of HMD and interactive controls can create a highly authentic experience which can accommodate extremely realistic representation of actual working sites. None of these features need take away from ID models and can be incorporated into existing ID models; decisions regarding the use of fidelity in the environment and experience can be made independently of the adoption of ID models.

The participants agreed that they were taking the scenarios 'more seriously' when using the HMD than they were in Second Life. This has important implications for supply chain focused education and for education or training for workers in operational sites, particularly if the way that participants respond to dangers in the virtual environment then transfers back to the real world.

Throughout the experiments it became clear that fluid interaction with the environment, the ability to easily perceive the world nearby, hearing sounds, and being able to see small but realistic visual elements (e.g., reflections on water), all helped create a compelling and realistic environment that enabled participants to feel 'detached' from the real world. This immersion in the high-fidelity virtual environment is related to a stronger sense of fascination with this environment and portrayal of the world, which should lead to stronger senses of intrinsic motivation. The implication is that the use of such high-fidelity environments in educational settings should engage students and help them to learn, when appropriately used in support of established ID models such as the four-part ID model proposed by Van Merriënboer et al. (2004).

In workplaces, it is important that prior training and experiences are used in a supportive fashion to enable firms to quickly and effectively train staff to deal with many operational challenges. A simple example is a 'site induction' which is used to educate a new worker (or a worker new to that specific site) about the dangers and risks on that site. There are a range of processes for this. In some states, passing a multi-choice quiz can enable the holder to gain a pass that enables them to access any construction site. Other firms use much more comprehensive site-tours guided by experienced individuals, which enable new staff to understand nuances and key areas of risk for them in the future. Somewhere in the middle would be the use of a videobased induction that shows actual dangers, but without the staff member 'being present'. Interestingly, our participants largely noted that their responsiveness to risks and dangers presented when using the HMD was greater than when dangers were presented using the low- 
fidelity Second Life. Scenarios were explored with 'more care and seriousness' when using the HMD in comparison to using Second Life. This strongly indicates that workplace inductions conducted using HMDs could present compelling engagement with risks and dangers while the participant is removed in time and space from the risks. We anticipate that the high-fidelity experience would transfer to subsequently treating the sources of risk and danger with greater care in the real environment at a later point in time.

Throughout this paper we have investigated the instructional design of a virtual and authentic learning environment. This was accomplished by using a series of experiments to understand the impact of the level of fidelity in the motivation and interest in the experience of the students. If highly authentic virtual environments can be created at low-cost this would enable more use of self-directed study with appropriate scaffolding.

We found that the use of the Oculus Rift HMD significantly increased how compelling participants found the experience and their ability to associate with the realism of the environment, creating a more engaging environment that participants were more interested in exploring. Their interactions with the environment also changed in a way that supports more effective learning if they were using the HMD-based scenarios. First, they treated the environment with greater caution and hesitation as they came to treat it with greater care. If this is strongly transferable to non-virtual environment it is an important outcome. Second, a range of sensory perceptions including physical feedback (such as from hitting a wall) and sound are important. Third, a more natural method of control within the environment would help participants to engage more readily, indicating a need to move away from keyboards/mouse as control mechanisms. Finally, participants felt very engaged in the environment and detached from the real-world when using the HMD.

Together, these findings indicate that the use of HMD can strongly support more authentic and high-fidelity virtual environments to support education. Does this require significant resourcing? Yes and no. Modern gaming environments, such as Unity 3D (which the Oculus Rift HMD can use) create many small visual details that improve fidelity (e.g., falling leaves); thus, while these environments require specialist skills to develop learning scenarios in, they offer greater fidelity than similar amounts of efforts in other, non-game-focused environments.

While these are important findings, it is important to note that the research involved a very small sample size which makes it difficult to generalise to the wider population. However, the high level of convergence indicates a strong effect size that could be empirically tested using a survey/questionnaire at a later point while using a modest sample size.

\section{Conclusions}

In this paper, we applied a preliminary study on the level of fidelity with respect to the immersion of the learner in an authentic environment. Despite the limited extend and sample size, the experiments and the conducted interviews with the participants showed that supply chain education can benefit by creating learning spaces that include emerging technology to immerse the learner beyond the traditional classroom or virtual learning environment. We find that a high level of fidelity is possible with effective game-based software (e.g., Unity 3D) coupled with state-of-the-art equipment such as HMDs and preferably a more intuitive interface for control. Competitive development and marketing of consumer technology caused a steep 
decline of cost for hardware which could not even considered for a general classroom just a few years ago; together, these technologies delivery a high-fidelity experience that motivates students. Our experiments showed the impact that perceptual immersion and engagement can be achieved with small investments - these tools can provide instructional designers with a simple toolkit to bring textbook content to life. We found that the elements important for a high-fidelity experience include visual and audio cues within the environment (provided by game-based software), interpersonal interactions (provided by other users in the environment or by intelligent bots), the ability to easily perceive the world around (provided by HMDs), to explore intuitively (provided by control technologies - an area of continuing development).

The experiments have demonstrated significant differences in the fidelity levels of different virtual environments when using different equipment and it has indicated some interesting areas for future research. First, a wider range of options would allow a more comprehensive evaluation of the impact of fidelity on experience. Similar experiments should also be conducted at higher levels of fidelity that leverage other control mechanisms to create a more fluid interaction with the environment. Tangling with wires may be avoided by using a mobile station that is secured to the back of the participants, leading to no tangle or confusion. These steps can be accomplished with relatively little investment. It will also be important to understand whether the 'seriousness' with which participants treated situations in the virtual environment also transfer back to real situations that they may experience in the real world.

Supply chain education involves evolving learner awareness regarding the complexity and multiple dimensions required to become productive and efficient addressing various topics from different disciplines (Wood and Reiners 2012). The multifaceted nature of real-world supply chains does not allow any kind of error as this could potentially cause millions of dollars in damage or trigger a chain of events that degrade the quality of life for the people, society, or the environment (Reiners et al. 2012). Scholars work hard to transfer the complexity of supply chains into textbooks and classrooms to support education. Textbooks embed learning materials using real-world case studies, theories are applied to real-world data, and results from optimisation algorithms for the problems in the warehouse location are visualised in mapoverlays; yet, despite these advances we still fail to achieve an authentic and immersive experience for the learner. Effective design of multiple classes and using a range of experiences can compensate for this (Reiners and Wood 2013). 'Study tours', an important method in contemporary tertiary education, are often used as an authenticity-increasing alternative to classroom-bound education. However, while "the study tour helps to bridge the gap between business theory and practice" (Porth 1997, 198), there is a significant drawback of study tours: they offer a limited peak into several 'slices' of the supply chain from an external perspective, limited to predefined walk-ways in safe areas and without access to deeper knowledge or data about the location; not to mention the high cost and risks involved in having learners at a dangerous site without specialised training (Hanna 2000).

\section{Acknowledgment}

Support for the production of this publication has been provided by the Australian Government Office for Learning and Teaching (Grant: Development of an authentic training environment to support skill acquisition in logistics and supply chain management, ID: ID122498). The views expressed in this publication do not necessarily reflect the views of the Australian Government Office for Learning and Teaching. 


\section{References}

Apostolopoulos, J.G., P.A. Chou, B. Culbertson, T. Kalker, M.D. Trott, and S. Wee. 2012. "The Road to Immersive Communication." Proceedings of the IEEE 100 (4): 974990. doi:10.1109/JPROC.2011.2182069.

Bastiaens, Theo J., and Rob L. Martens. 2000. "Conditions for Web-based Learning with Real Events." In Instructional and Cognitive Impacts of Web-Based Education, edited by Beverly Abbey, 1-31. Hershey, PA: IGI Global. http://www.igiglobal.com/chapter/conditions-web-based-learning-real/23896.

Biocca, F., and B. Delaney. 1995. "Immersive Virtual Reality Technology." In Communication in the Age of Virtual Reality, edited by F. Biocca and M. Levy, 57124. Hillsdale, NJ: Erlbaum.

Dede, Chris. 2009. "Immersive Interfaces for Engagement and Learning." Science 323 (5910) (February 1): 66-69. doi:10.1126/science.1167311.

DeFanti, Thomas A., Gregory Dawe, Daniel J. Sandin, Jurgen P. Schulze, Peter Otto, Javier Girado, Falko Kuester, Larry Smarr, and Ramesh Rao. 2009. "The StarCAVE, a Third-generation CAVE and Virtual Reality OptIPortal." Future Generation Computer Systems 25 (2) (February): 169-178. doi:10.1016/j.future.2008.07.015.

Deimann, Markus, and Theo Bastiaens. 2010. "Competency-based Education in an Electronic-supported Environment: An Example from a Distance Teaching University." International Journal of Continuing Engineering Education and LifeLong Learning 20 (3/4/5): 278-289. doi:10.1504/IJCEELL.2010.037046.

Gulikers, Judith T. M., Theo J. Bastiaens, and Rob L. Martens. 2005. "The Surplus Value of an Authentic Learning Environment." Computers in Human Behavior 21 (3): 509521. doi:10.1016/j.chb.2004.10.028.

Hanna, Mark D. 2000. "Touring a Supply Chain with Students: Pedagogical and Practical Considerations." Production and Operations Management 9: 203-211. doi:10.1111/j.1937-5956.2000.tb00334.x.

Klieme, Eckhard, and Detlev Leutner. 2006. "Kompetenzmodelle Zur Erfassung Individueller Lernergebnisse Und Zur Bilanzierung von Bildungsprozessen. Beschreibung Eines Neu Eingerichteten Schwerpunktprogramms Der DFG." Zeitschrift Fuer Paedagogik 52 (6): 876-903.

Merrill, M. David. 2002. "First Principles of Instruction." Educational Technology Research and Development 50 (3): 43-59. doi:10.2307/30220335.

Oculus. 2013. "About Oculus VR.” http://www.oculusvr.com/company/.

Palmer, F. 1995. "Interpersonal Communication and Virtual Reality: Mediating Interpersonal Relationships." In Communication in the Age of Virtual Reality, edited by F. Biocca and M. Levy, 277-302. Hillsdale, NJ: Erlbaum.

Porth, Stephen J. 1997. "Management Education Goes International: A Model for Designing and Teaching a Study Tour Course." Journal of Management Education 21 (May 1): 190-199. doi:10.1177/105256299702100204.

Reiners, Torsten, and Lincoln C. Wood. 2013. "Immersive Virtual Environments to Facilitate Authentic Education in Logistics and Supply Chain Management." In Learning Management Systems and Instructional Design: Best Practices in Online Education, edited by Yefim Kats, 323-343. Hershey, PA: IGI Global. 10.4018/978-14666-3930-0.

Reiners, Torsten, Lincoln C. Wood, and Sue Gregory. 2014. "Experimental Study on Technology-induced Authentic Immersion in Virtual Worlds." School of Information Systems Working Paper Series. Perth, Australia: Curtin University.

Van Merriënboer, Jeroen J. G. 1997. Training Complex Cognitive Skills: A FourComponent Instructional Design Model for Technical Training. Englewood Cliffs, NJ: Educational Technology Publications.

Van Merriënboer, Jeroen J. G., Theo Bastiaens, and Albert Hoogveld. 2004. "Instructional Design for Integrated E-learning." In Integrated E-Learning: Implications for 
Pedagogy, Technology and Organization, edited by Wim Jochems, Jeroen J. G. van Merriënboer, and Rob Koper, 13-23. London, UK: RoutledgeFalmer.

Wood, Lincoln C., and Hendrik Reefke. 2010. "Working with a Diverse Class: Reflections on the Role of Team Teaching, Teaching Tools and Technological Support." In IADIS International Conference on International Higher Education (IHE 2010), 72-79. Perth, Australia: IADIS Press.

Wood, Lincoln C., and Torsten Reiners. 2012. "Gamification in Logistics and Supply Chain Education: Extending Active Learning." In IADIS International Conference on Internet Technologies \& Society 2012, edited by Piet Kommers, Tomayess Issa, and Pedro Isaías, 101-108. Perth, Australia: IADIS Press. 